\title{
Article
}

\section{In-Situ Evaluation of the Pitch of a Reflective-Type Scale Grating by Using a Mode-Locked Femtosecond Laser}

\author{
Dong Wook Shin ${ }^{1}$, Lue Quan ${ }^{1}$, Yuki Shimizu ${ }^{1, *}$, , Hiraku Matsukuma ${ }^{1}$, Yindi Cai ${ }^{1,2}{ }^{\circledR}$, Eberhard Manske ${ }^{3}$ \\ and Wei Gao ${ }^{1}$ \\ 1 Precision Nanometrology Laboratory, Department of Finemechanics, Tohoku University, Sendai 980-8579, Japan; \\ shin.dong.wook.t6@dc.tohoku.ac.jp (D.W.S.); quan.lue.p3@dc.tohoku.ac.jp (L.Q.); \\ hiraku.matsukuma.d3@tohoku.ac.jp (H.M.); caiyd@dlut.edu.cn (Y.C.); i.ko.c2@tohoku.ac.jp (W.G.) \\ 2 Key Laboratory for Micro/Nano Technology and System of Liaoning Province, Dalian University of \\ Technology, Dalian 116024, China \\ 3 Department of Mechanical Engineering, Ilmenau University of Technology, 98693 Ilmenau, Germany; \\ Eberhard.Manske@tu-ilmenau.de \\ * Correspondence: yuki.shimizu.d2@tohoku.ac.jp; Tel.: +81-22-795-6950
}

check for updates

Citation: Shin, D.W.; Quan, L.; Shimizu, Y.; Matsukuma, H.; Cai, Y.; Manske, E.; Gao, W. In-Situ

Evaluation of the Pitch of a Reflective-Type Scale Grating by Using a Mode-Locked Femtosecond Laser. Appl. Sci. 2021, 11, 8028. https://doi.org/10.3390/ app11178028

Academic Editor: Andreas Fischer

Received: 27 July 2021

Accepted: 28 August 2021

Published: 30 August 2021

Publisher's Note: MDPI stays neutral with regard to jurisdictional claims in published maps and institutional affiliations.

Copyright: (c) 2021 by the authors. Licensee MDPI, Basel, Switzerland. This article is an open access article distributed under the terms and conditions of the Creative Commons Attribution (CC BY) license (https:/ / creativecommons.org/licenses/by/ $4.0 /)$.

\begin{abstract}
Major modifications are made to the setup and signal processing of the method of in-situ measurement of the pitch of a diffraction grating based on the angles of diffraction of the diffracted optical frequency comb laser emanated from the grating. In the method, the improvement of the uncertainty of in-situ pitch measurement can be expected since every mode in the diffracted optical frequency comb laser can be utilized. Instead of employing a Fabry-Pérot etalon for the separation of the neighboring modes in the group of the diffracted laser beams, the weight-of-mass method is introduced in the method to detect the light wavelength in the Littrow configuration. An attempt is also made to reduce the influence of the non-uniform spectrum of the optical comb laser employed in the setup through normalization operation. In addition, an optical alignment technique with the employment of a retroreflector is introduced for the precise alignment of optical components in the setup. Furthermore, a mathematical model of the pitch measurement by the proposed method is established, and theoretical analysis on the uncertainty of pitch measurement is carried out based on the guide to the expression of uncertainty in measurement (GUM).
\end{abstract}

Keywords: diffraction grating; grating pitch; mode-locked femtosecond laser; laser diffraction; diffraction equation; measurement uncertainty analysis

\section{Introduction}

A diffraction grating, which has periodic fine pattern structures on its surface, is one of the most important optical components often employed in many scientific and industrial fields. A diffraction grating can be employed as the scale for measurement in an optical encoder, in which the relative displacement between an optical head and a diffraction grating can be measured [1]. Since the fine pattern structures on a scale grating are employed as the graduations for measurement [2,3], the evaluation of the grating pitch is an important task to assure the performance of an optical encoder.

Many methods have been developed so far to evaluate the grating pitch of a scale grating. The observation of three-dimensional profiles of fine pattern structures on a scale grating by high-resolution measuring instruments such as critical-dimension (CD) scanning electron microscopes (SEMs) or atomic force microscopes (AFMs) is a direct and straightforward method [4-7]. However, this method is not suitable for the evaluation of the whole length of a scale grating due to the limited measurement throughput. On the other hand, a method utilizing the laser diffraction, in which the period of pattern structure can be evaluated by the angle of diffraction of a diffracted laser beams emanated from the grating surface under evaluation, is a promising one for the evaluation of the whole 
length of a scale grating [8-13]. Although the measurand capable of being evaluated in this method is limited to a mean of the grating period over the area where the measurement laser beam is irradiated (namely, this method is an indirect method for measurement of the pitch of a scale grating), it is suitable for the calibration of large area grating patterns due to its high measurement throughput and non-contact measurement apparatus. On the other hand, in the conventional methods based on the laser diffraction with a single-mode measurement laser beam, the information to be obtained in experiments are quite limited due to the nature of the laser diffraction; in most of the cases, a few-order diffracted beams can be obtained when projecting a measurement laser beam onto a scale grating. This limits the measurement accuracy of a grating pitch.

To address the aforementioned issues, a new concept for in-situ [14] pitch measurement employing an optical frequency comb laser has been proposed. An optical frequency comb laser contains a lot of modes equally spaced in the optical frequency domain [15]. Since each mode has a deterministic light wavelength, a group of the first-order diffracted beams having different angles of diffraction can be obtained. With the employment of a fiber detector and a spectrometer for the observation of the diffracted beams in the optical frequency domain, much more information can be obtained for the pitch evaluation based on laser diffraction. Stabile and accurate optical frequency of each optical mode in an optical frequency comb [16] is also expected to contribute to the accuracy improvement of the in-situ pitch measurement. The feasibility of the proposed concept has been verified through some experiments with a developed prototype setup [17]. However, there are some problems that need to be addressed for applying optical frequency comb as a light source for the laser diffraction method. Since an optical frequency comb has multiple frequency modes with a narrow spacing in the optical frequency domain, the neighboring diffracted beams overlap with each other, resulting in the difficulty of identifying each mode in a spectrometer [18]. In the previous study by the authors [17], a Fabry-Pérot etalon with a high free-spectral range was employed to expand the mode-spacing of the optical frequency comb so that each mode in the group of the first-order diffracted beams could be distinguished. However, this could reduce the information of the angles of diffraction of the diffracted beams, resulting in diminishing the benefit of the proposed concept. In addition, the misalignments of the measurement laser beam and a fiber detector composed of an objective lens and a single-mode fiber in the setup could be sources of uncertainty in measurement of the grating pitch. Theoretical investigation on the measurement uncertainty of the proposed concept is thus necessary while considering the influences of these optical misalignments, although it has remained a task to be addressed.

In this paper, following the previous study by the authors [17], a major modification is made to the prototype setup by removing a Fabry-Pérot etalon, while applying a new signal processing technique based on the weight of mass method to the detection of a peak wavelength for measurement of the grating pitch. A mathematical model of the pitch measurement is also established based on the modified setup by including some parameters related to optical misalignments. By using the established mathematical model, a measurement uncertainty analysis is carried out based on the guide to the expression of uncertainty in measurement (GUM). It should be noted that, regarding the concept of the in-situ pitch measurement, all the experiments described in this paper have been carried out in an ordinary laboratory room condition where the optical setup is not shielded from external disturbances such as illumination with fluorescent lights.

\section{Methods for In-Situ Measurement of the Grating Pitch with a Diffracted Optical Frequency Comb Laser}

Methods for measurement of the grating pitch with diffracted laser beams are based on the light diffraction equation that can be expressed as follows [19]:

$$
P \sin \theta_{\text {in }}+P \sin \theta_{\text {diff }}=m \lambda
$$


where $P$ is the grating pitch, $\theta_{\text {in }}$ is the angle of incidence of the measurement laser beam, $\theta_{\text {diff }}$ is the angle of diffraction of the $m$ th-order diffracted beam, and $\lambda$ is the light wavelength of the measurement laser beam. It should be noted that $\theta_{\text {in }}$ and $\theta_{\text {diff }}$ are defined with respect to the normal of the grating surface. Figure 1a shows a schematic of the setup for measurement of the pitch deviation of a grating. The setup is mainly composed of a monochromatic laser source, a rotary table equipped with a high-precision rotary encoder, and a detector unit (not indicated in the figure). A scale grating can be mounted on the rotary table for pitch measurement. In the setup, a collimated laser beam from the laser source is projected onto the grating surface to generate diffracted beams. The configuration where $\theta_{\text {in }}$ becomes equal to $\theta_{\text {diff }}$, as shown in Figure $1 \mathrm{a}$, is referred to as the Littrow configuration [20]. Under the condition of the $m$ th-order Littrow configuration with the Littrow angle $\theta_{m}, \theta_{\text {in }}=\theta_{\text {diff }}=\theta_{m}$, and the following equation can be obtained from Equation (1):

$$
P=\frac{m \lambda}{2 \sin \theta_{m}}
$$

(a)

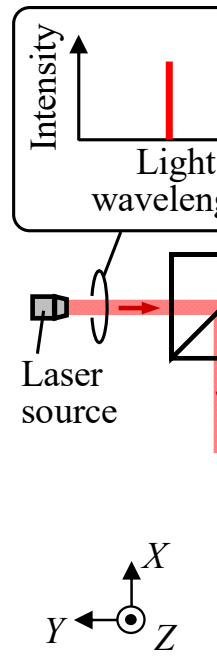

(b)

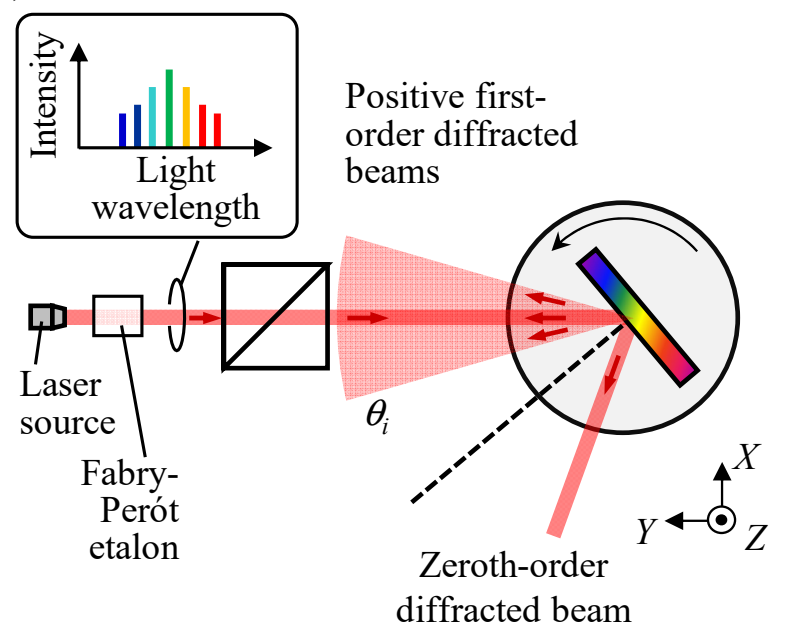

Figure 1. Laser diffraction method for measurement of a grating pitch: (a) Conventional method with a single-mode laser; (b) Proposed method with a mode-locked laser.

According to the above equation, the parameters need to be obtained for the calculation of $P, \theta_{m}$ and $\lambda$. Since $\lambda$ can be treated as a known parameter, the grating pitch $P$ can be evaluated by measuring $\theta_{m}$ in experiments. By rotating the grating under inspection with a rotary table equipped with a high-precision rotary encoder, multiple diffracted beams can be observed. For the detection of diffracted beams, image sensors such as a charge-coupled device (CCD) are often employed in the detector unit. In most cases, the number of diffracted beams available for pitch measurement is quite limited when employing a monochromatic laser source. For example, in the case where a scale grating having a nominal pitch of $1000 \mathrm{~nm}$ is evaluated with a laser beam having a light wavelength of $633 \mathrm{~nm}$, the maximum $m$ becomes 3; namely, only six diffracted beams (three positive diffracted beams and three negative diffracted beams) can be obtained. It should also be noted that the area to be irradiated by the measurement laser beam could vary with the increase of $m$. Namely, the grating pitch $P$ evaluated by the diffracted beams with different diffraction orders could be from a different area on the scale grating.

These issues can be addressed by the method with an optical frequency comb laser [17]. Figure $1 \mathrm{~b}$ shows a schematic of the optical setup for pitch measurement with an optical frequency comb laser. When the collimated laser beam of an optical frequency comb is projected onto a scale grating, the group of the positive first-order diffracted beams emanating from the projected area can be obtained [21]. It should be noted that the group 
of the negative first-order diffracted beams and those of the higher-order diffracted beams are not indicated in the figure for the sake of simplicity. The Littrow angle $\theta_{m, i}$ of the $i$ th mode in the $m$ th-order diffracted beam can be expressed by the following equation:

$$
P_{m, i}=\frac{m}{2 \sin \theta_{(m, i)}} \times \frac{c}{v_{i}}=\frac{m}{2 \sin \theta_{(m, i)}} \times \frac{c}{i \times v_{r e p}+v_{C E O}}
$$

where $v_{i}$ is the optical frequency of the ith mode, which can be represented by using the pulse repetition frequency $v_{\text {rep }}$ and the carrier offset frequency $v_{\text {CEO }}$ as $v_{i}=i \cdot v_{\text {rep }}+v_{\text {CEO }}$. Since a lot of Littrow angles can be obtained in the same diffraction order, the improvement of the measurement uncertainty with the averaging effect can be expected.

It should be noted that the diffracted laser beams should be observed in the optical frequency domain so that the light wavelength of each mode in the Littrow configuration can be determined. In the previous work by the authors [17], a Fabry-Pérot etalon with a high free-spectral range was employed so that each mode could be distinguished in the optical frequency domain. In this paper, a major modification is made to the optical setup; instead of separating the modes with the Fabry-Pérot etalon, a single-mode optical fiber was placed on the optical axis to specify the light wavelength mode corresponding to the Littrow configuration from the overlapped neighboring modes as shown in Figure 2. When a group of the diffracted beams was made incident to the optical fiber, the beams propagated through the core of the optical fiber by reflection on the boundary surface with the shelter part named clad. Due to the loss of the intensity from the reflection, the intensity spectrum consequently shows Gaussian distribution in which the light mode made parallelly incident to the optical axis shows the highest intensity. The spectrum of propagated beams within the mode field diameter (MFD), determined by the fall of the intensity to $\mathrm{e}^{-2}$, was then detected by the optical spectrum analyzer, and the peak wavelength was determined by the weight of mass method. This modification enables the setup to fully utilize the information of the modes in the group of the diffracted beams.

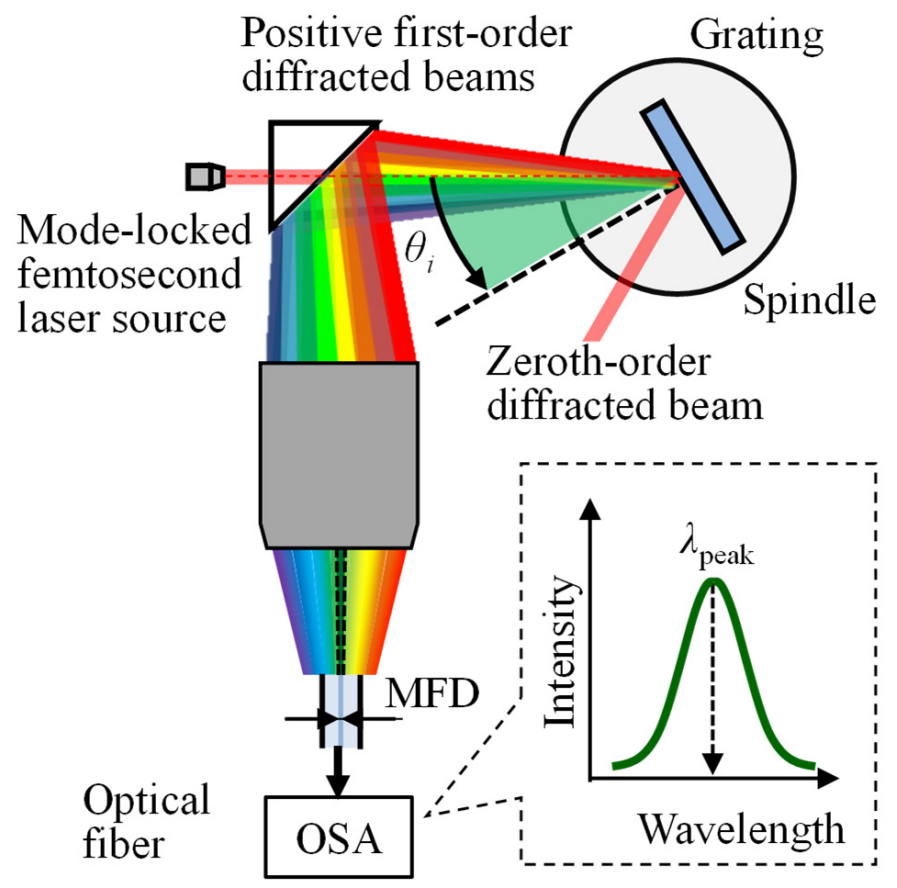

Figure 2. Peak wavelength detection for measurement of the grating pitch.

Figure 3a shows a three-dimensional drawing of the setup shown in Figure 1b. With the employment of a polarizing beam splitter (PBS) and a quarter-wave plate (QWP), the diffracted beams can be guided to the detector unit without going back to the laser source. The detector unit consists of an objective lens and a single-mode fiber, one side of 
which is connected to an optical spectrum analyzer (OSA). In the setup, the normal of the grating is aligned to be parallel with the $X$-axis when the angular position of the spindle is set to $\theta=0^{\circ}$. However, the measurement laser beam and the detector unit have angular misalignments with respect to the optical axis as shown in Figure $3 b$. Thus, variable $\alpha$ is given to the angular misalignment of the measurement laser beam about the $Z$-axis with respect to the $X$-axis, and variable $\beta$ is given to that of the optical axis of the detector unit about the $X$-axis with respect to the $Z$-axis. Regarding these misalignments in the setup, the diffraction equation in Equation (2) for the first-order diffracted beams can be modified as follows:

$$
\begin{aligned}
& P_{\text {pos } \_}=\frac{\lambda}{\sin \theta_{\text {in }}+\sin \theta_{\text {diff }}}=\frac{\lambda}{\sin \left(\Delta \theta_{+1}-\frac{\alpha}{2}\right)+\sin \left(\Delta \theta_{+1}+\frac{\alpha}{2}+\beta\right)}=\frac{\lambda}{2 \cos \left(\frac{\alpha-\beta}{2}\right) \sin \left(\Delta \theta_{+1}+\frac{\beta}{2}\right)} \\
& P_{\text {neg } i}=\frac{\lambda}{\sin \theta_{\text {in }}+\sin \theta_{\text {diff }}}=\frac{\lambda}{\sin \left(\Delta \theta_{-1}+\frac{\alpha}{2}\right)+\sin \left(\Delta \theta_{-1}-\frac{\alpha}{2}-\beta\right)}=\frac{\lambda}{2 \cos \left(\frac{\alpha-\beta}{2}\right) \sin \left(\Delta \theta_{-1}-\frac{\beta}{2}\right)}
\end{aligned}
$$

where $\lambda$ is the wavelength of the measurement beam, $\Delta \theta_{ \pm 1}$ is the angular position of the spindle, $\alpha$ is the angular misalignment of the measurement laser beam about the $Z$-axis with respect to the $X$-axis, and $\beta$ is the angular misalignment of the optical axis of the detector unit about the $X$-axis with respect to the $Z$-axis.

(a)

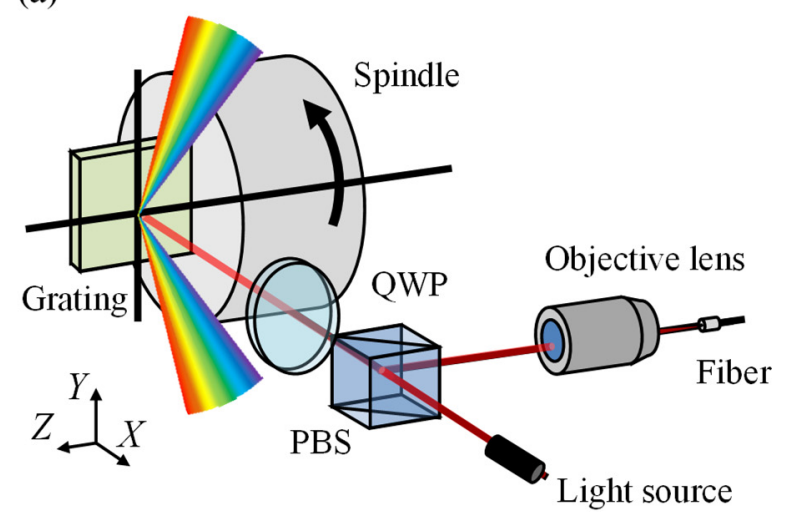

(b)

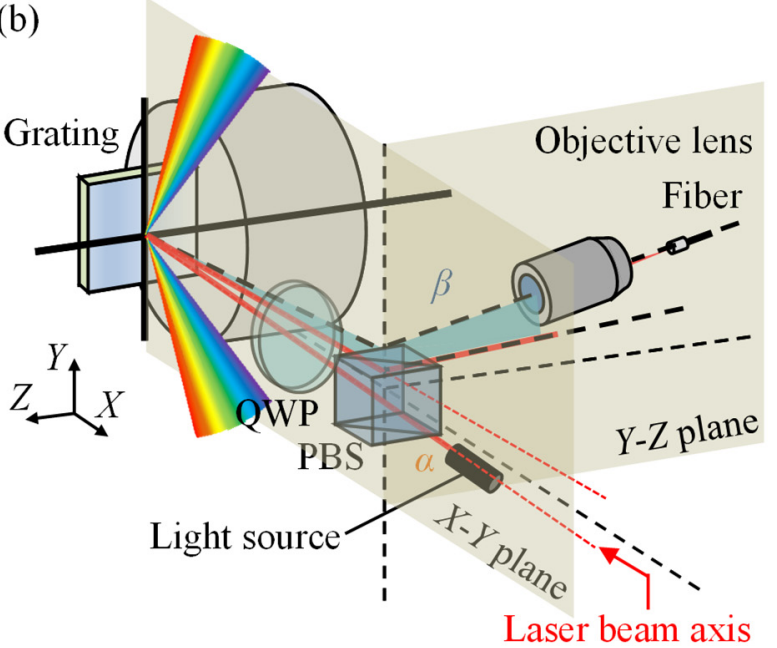

Figure 3. Optical configuration for measurement of the grating pitch; (a) Without the angular misalignments; (b) With the angular misalignments.

Based on Equations (4) and (5), the influences of $\alpha$ and $\beta$ on measurement of the pitch deviation are estimated quantitatively. Figure $4 a, b$ show the difference between the calculated pitch $P_{\mathrm{c}}$ obtained based on Equations (4) and (5) and the nominal pitch $P$ due to the angular misalignments $\alpha$ and $\beta$, respectively. Calculations are carried out under the condition of $P=833.33 \mathrm{~nm}$ and an angular position of the spindle of $28^{\circ}$. The influence of $\alpha$ is estimated to be approximately $-32 \mathrm{pm}$ under the condition of $\alpha=1^{\circ}$, while that of $\beta$ is estimated to be approximately $13.7 \mathrm{~nm}$ under the condition of $\beta=1^{\circ}$. From these results, it can be concluded that the corrections of $\alpha$ and $\beta$ are necessary for accurate pitch measurement. 
(a)

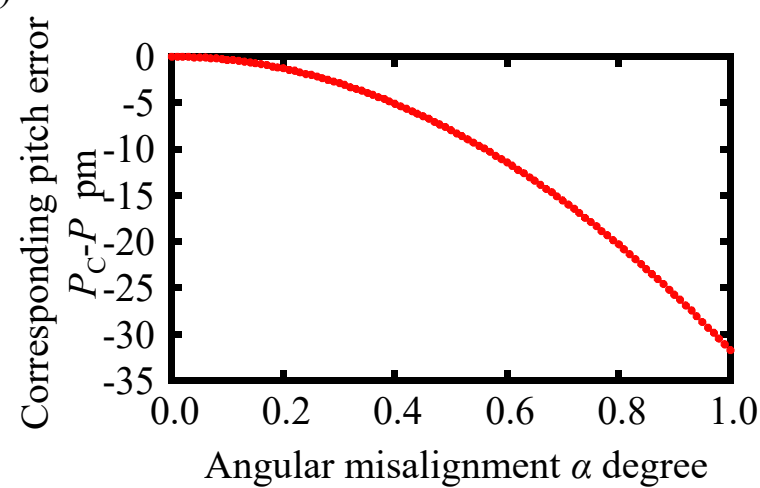

(b)

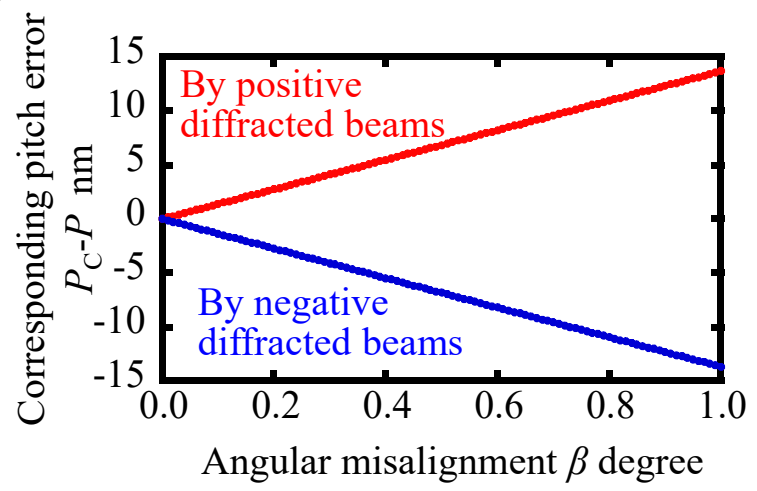

Figure 4. Influence of misalignments in the setup; (a) Influence of the angular misalignment of the measurement laser beam about the Z-axis $(\alpha)$; (b) Influence of the angular misalignment of the measurement laser beam about the $X$-axis $(\beta)$.

\section{Design and Development of the Optical Setup and Verification Experiment}

A schematic of the setup employed in the following experiments is shown in Figure 5a. A fiber-based mode-locked femtosecond laser with a pulse repetition frequency $v_{\text {rep }}$ of $100 \mathrm{MHz}$ was employed as the laser source. The laser beam from the laser source was guided into the setup by using an optical fiber. The laser beam from the edge of the fiber was collimated by a collimating lens, and was then projected onto a grating mounted on a spindle. It should be noted that a half-wave plate was placed just after the collimating lens in the optical path of the laser beam so that the polarization direction of the laser beam could be controlled. A pair of a quarter-wave plate and a polarizing beam splitter was employed as an optical isolator to prevent the diffracted beam from going back to the laser source. The diffracted laser beams, whose propagating directions were changed by the polarizing beam splitters, were captured by a detector unit composed of an objective lens and a single-mode fiber with a mode-field diameter of approximately $10.8 \mu \mathrm{m}$. One end of the single-mode fiber was placed at the focal plane of the objective lens, while the other was connected to a commercial optical spectrum analyzer (AQ6370D, Yokogawa Co., Ltd., Tokyo, Japan) so that the captured diffracted laser beams could be analyzed in the optical frequency domain. Figure $5 \mathrm{~b}$ shows a photograph of the setup employed in the following experiments.

(a)

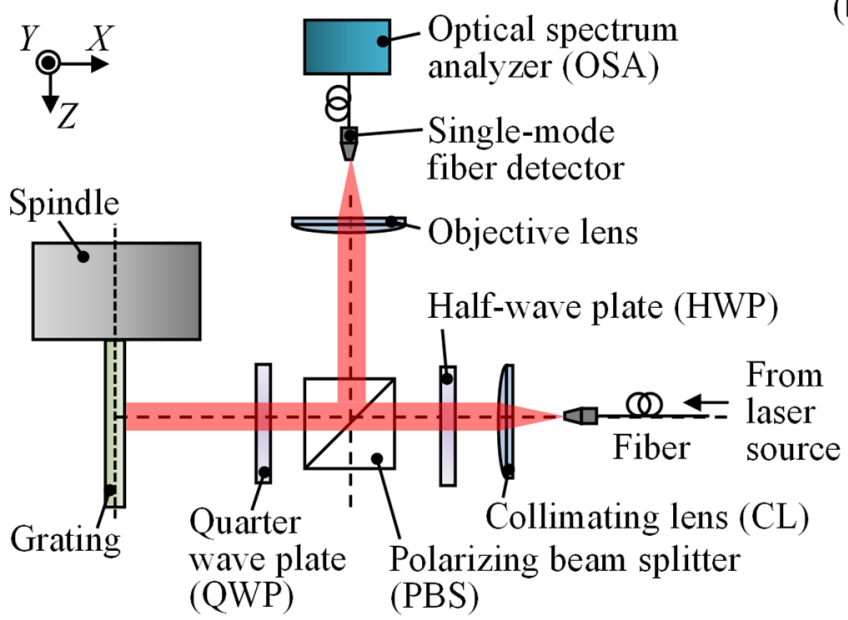

(b)

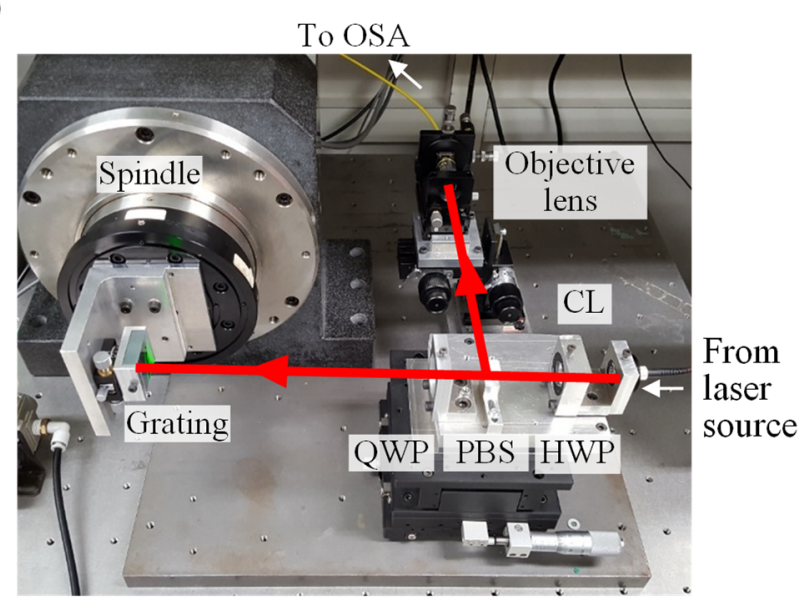

Figure 5. Experimental setup for measurement of the pitch of a reflective-type diffraction grating: (a) Schematic of the setup; (b) A photograph of the setup.

The detection of the angular position of the grating in the developed setup could strongly affect the pitch measurement by the proposed method. Therefore, at the beginning 
of the following experiments, the stability of the reading of the rotary encoder embedded in the spindle in the developed setup was evaluated. A variation of the rotary encoder reading was observed while the spindle was kept stationary at the positive and negative first-order Littrow configurations. Figure $6 \mathrm{a}, \mathrm{b}$ show the variations of the rotary encoder reading at the angular positions $\theta=28.08^{\circ}$ and $-28.08^{\circ}$, respectively, in a period of two hours. A sampling frequency of the encoder reading was set to be $10 \mathrm{~Hz}$. Standard deviations of the rotary encoder reading were evaluated to be 0.06054 arc-second and 0.06475 arc-second for the positive and negative Littrow configurations, respectively. From these results, the spindle was confirmed to have positioning stability within the specification of the spindle (1 arc-second).

(a)

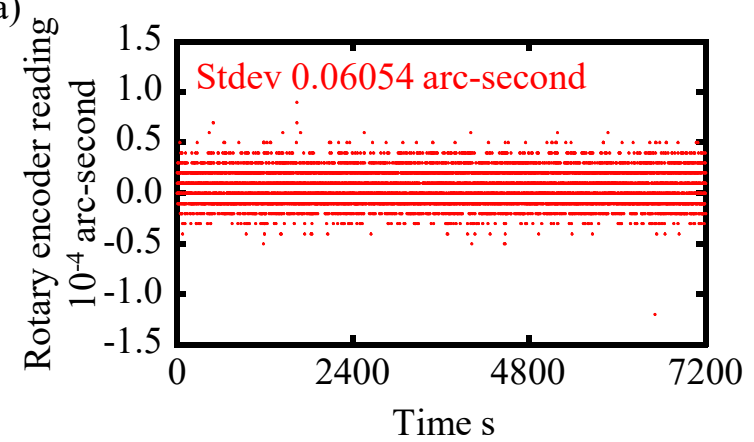

(b)

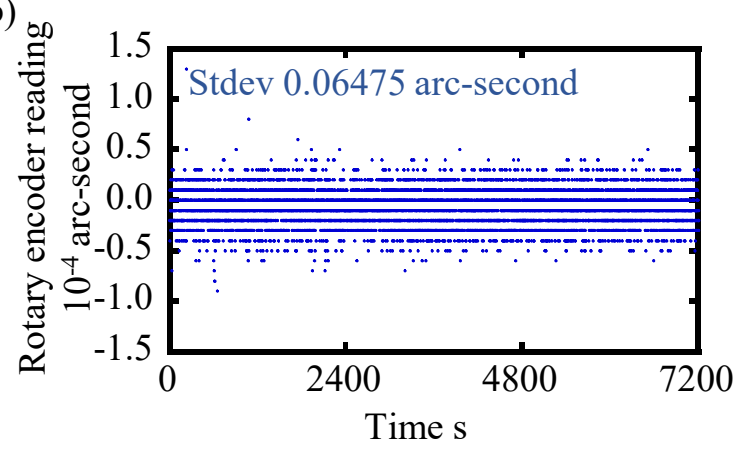

Figure 6. Reading of the rotary encoder embedded to the air-bearing spindle: (a) Positive first-order Littrow configuration; (b) Negative First-order Littrow configuration.

Alignments of the optical setup can be carried out by using the zeroth and first-order diffracted beams. Figure 7a shows a schematic of the setup. A retroreflector, which has the ability to reflect the laser beam to its source, is placed in front of the scale grating, while a beam profiler is placed at the focal plane of the objective lens. The reflected measurement laser beam is then focused on the detector plane at a point $P_{M}$ in the beam profiler, and its $Y$-position is recorded. After that, the retroreflector is removed from the setup where the angular position of the spindle is set to $\theta=0^{\circ}$; this operation makes the zeroth-order diffracted beam from the scale grating be focused on the beam profiler at a point $P_{0}$. Meanwhile, with the existence of the angular misalignment $\alpha$, the focused laser beam is made to shift along the $Y$-direction. According to the principle of laser autocollimation [22], the $Y$-directional spot displacement $\Delta d$ on the beam profiler due to $\alpha$ can be expressed by the following equation:

$$
\Delta d=F \tan \alpha
$$

Since the focal length $F$ of the objective lens was known to be $31 \mathrm{~mm}, \alpha$ can be evaluated by detecting $\Delta d$ with the beam profiler.

(a)

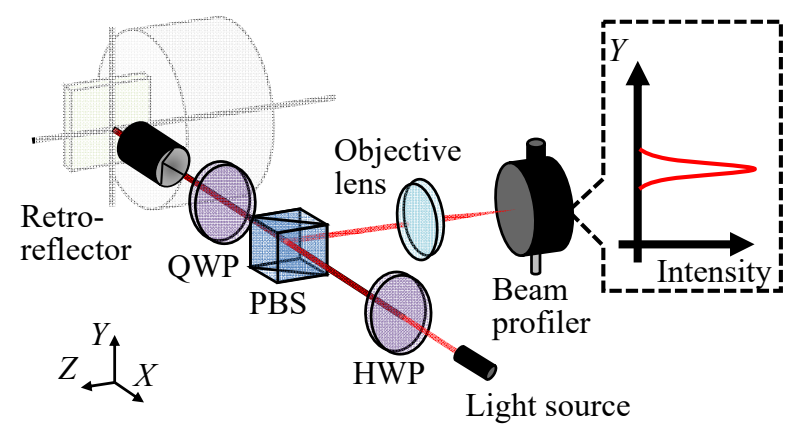

(b) $\underline{\text { Step } 1}$

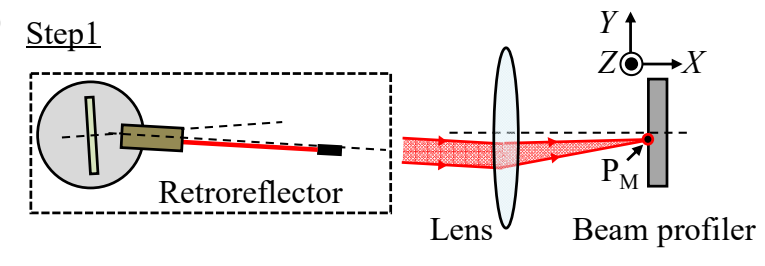

(c)

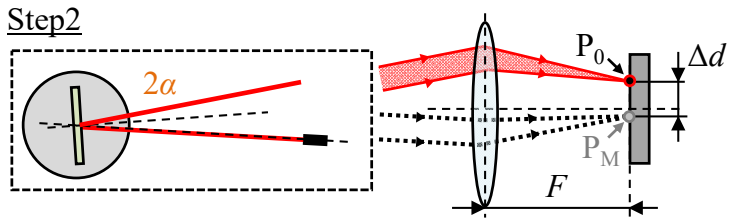

Figure 7. Alignment of the measurement laser beam by using a retroreflector and a beam profiler: (a) Setup with a retroreflector; $(\mathbf{b}, \mathbf{c})$ Procedure of the alignment. 
Figure 8 shows the variations of the $Y$-positions of the measurement laser beam and the zeroth-order diffracted beam focused on the beam profiler in a period of $20 \mathrm{~s}$. As the target grating, a holographic-type grating with a line density of $1200 / \mathrm{mm}$ was employed. From the mean values, $\Delta d$ was evaluated to be $1.143 \mu \mathrm{m}$, and $\alpha$ was thus evaluated to be 7.579 arc-seconds based on Equation (6).

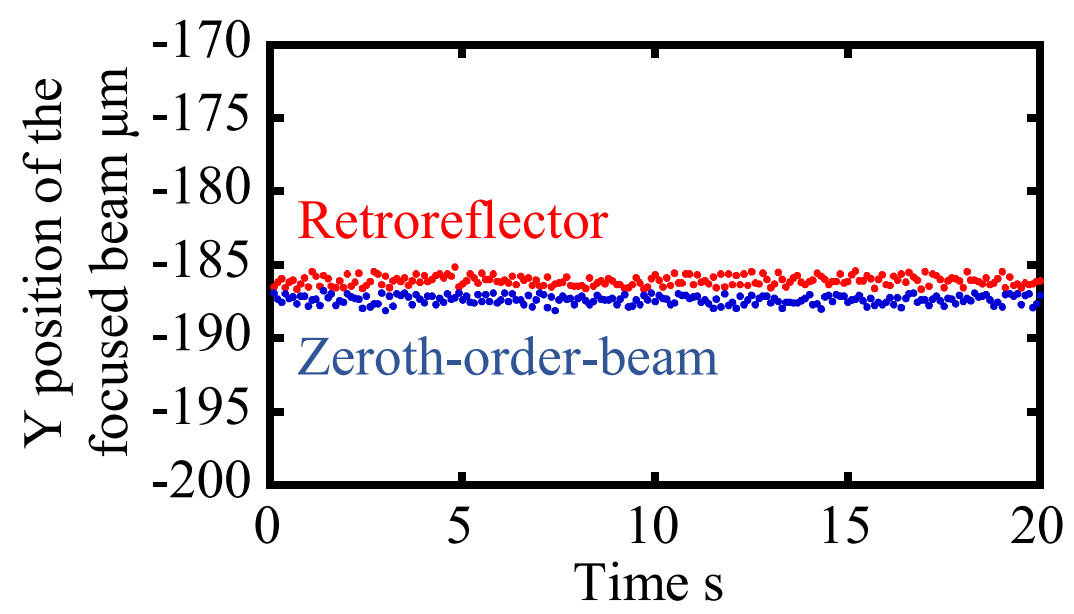

Figure 8. $Y$-position of the focused laser beam on the beam profiler during the alignment.

After the verification of the angular misalignment $\alpha$, the alignment of the single-mode fiber in the fiber detector unit was carried out while the angular position of the spindle was kept stationary at $\theta=0^{\circ}$. Figure 9 shows the spectrum of the zeroth-order diffracted beam captured by the detector unit. The alignment of the single-mode fiber was carried out so that the total laser power to be detected by the detector unit became maximum while observing the spectrum of the captured laser beam. As can be seen in the figure, a non-uniform spectrum of the optical frequency comb laser was successfully observed.

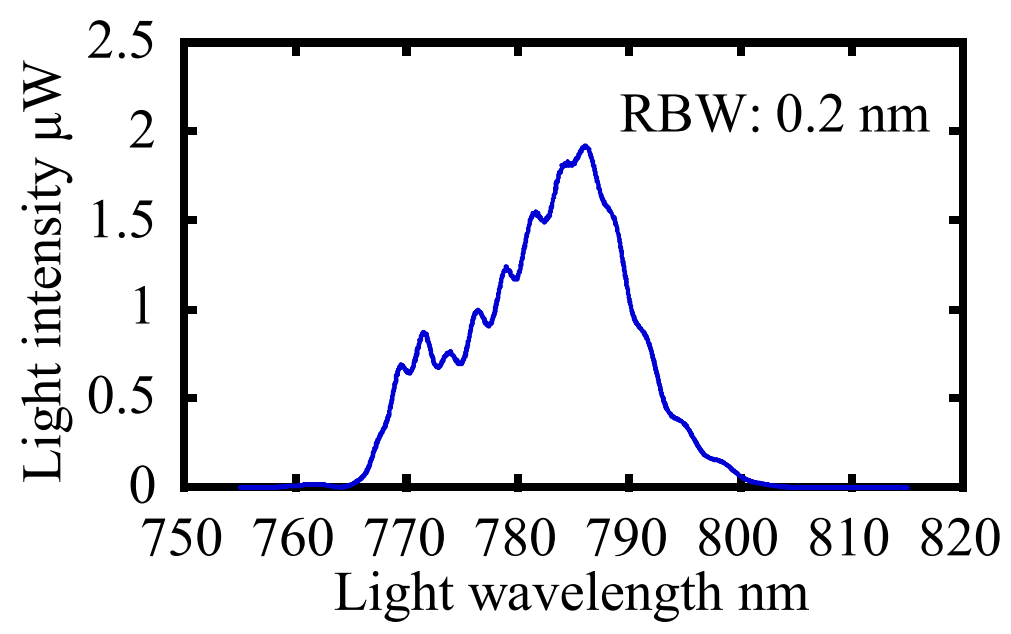

Figure 9. The optical spectrum of the mode-locked femtosecond laser employed in this paper.

Meanwhile, the detector unit had a small angular misalignment $\beta$ about the $X$-axis even after the alignment; this can be evaluated in experiments by using the positive and negative first-order diffracted beams. Figure 10a,b show the variations of the spectra of the positive and negative first-order diffracted beams captured by the same detector unit when rotating the scale grating. In the figures, the spectra obtained at the angular position $|\theta|$ ranging from $27.48^{\circ}$ to $27.52^{\circ}$ in a step of $0.01^{\circ}$ are plotted. As can be seen in the figure, the spectra of the negative first-order diffracted beams were found to shift 
toward the shorter light wavelength; this was mainly due to the influence of $\beta$, regarding Equations (4) and (5).

(a)

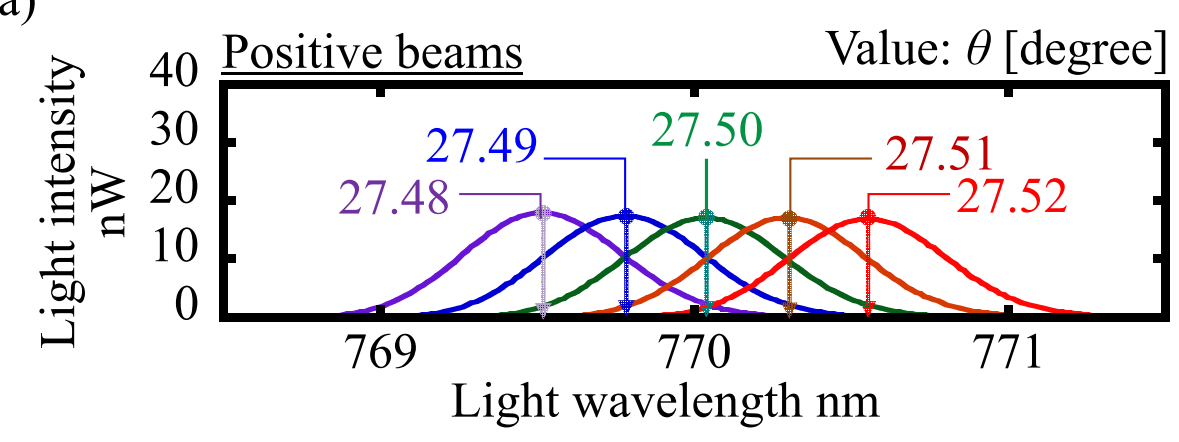

(b)

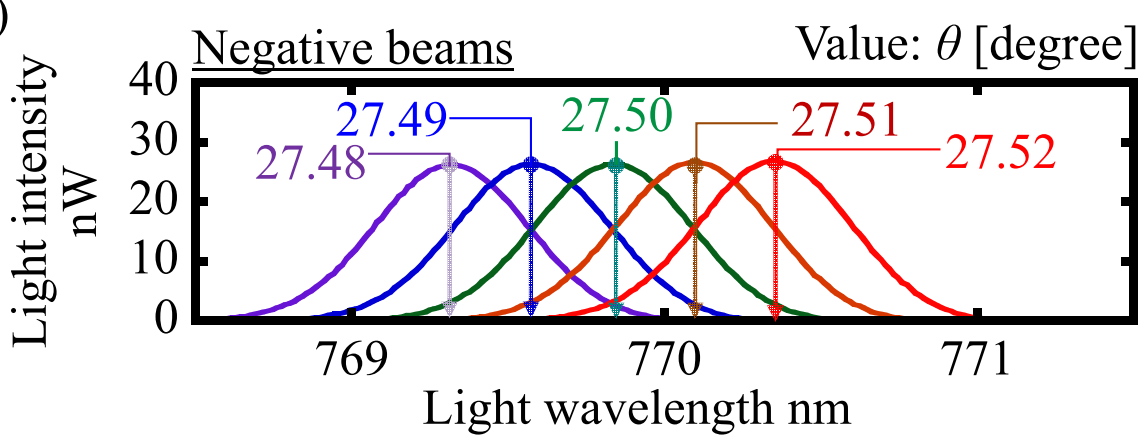

Figure 10. Spectra obtained at each angular position of the scale grating: (a) with the positive first-order diffracted beams; (b) with the negative first-order diffracted beams.

The grating pitch $P$ can be evaluated by detecting the peak wavelength of the spectrum at each angular position of the spindle $\theta$. In this paper, a weight-of-mass method based on the following equation was employed to detect the peak wavelength $\lambda_{\text {center }}$ in a spectrum:

$$
\lambda_{\text {Center }}=\frac{\sum\left(I_{\lambda_{i}} \times \lambda_{i}\right)}{\sum I_{\lambda_{i}}}
$$

where $\lambda_{i}$ is the $i$ th sampled wavelength, and $I_{\lambda i}$ is the light intensity of the light wavelength component $\lambda_{i}$. Figure 11a shows the grating pitch calculated based on Equations (4) and (5) by the series of $\lambda_{\text {center }}$ obtained at different angular positions $\theta$ of the spindle. It should be noted that the parameter $\beta$ was set to be $0^{\circ}$. As can be seen in the figure, a discrepancy mainly due to the misalignment $\beta$ was found between the grating pitches $P_{\text {pos } \_i}$ and $P_{\text {neg_i } i}$ obtained from the positive and negative first-order diffracted beams, respectively. To compensate for the influence of $\beta$, calculations based on Equations (4) and (5) were repeated by changing $\beta$ in a step of $0.0001^{\circ}$. Figure $11 \mathrm{~b}$ shows the sum of $\left|P_{\text {pos }_{-} i}-P_{\text {neg }_{-} i}\right|$ at each $\beta$. By performing fitting by sixth-order function approximation and finding the extremum, $\beta$ that minimizes $\Sigma\left|P_{\text {pos } \_i}-P_{\text {neg } \_i}\right|$ was evaluated to be $0.001212^{\circ}$ (4.363 arc-seconds). Figure 11c shows $P_{\text {pos } \_i}$ and $P_{\text {neg } \_i} i$ calculated based on Equations (4) and (5) under the condition of $\beta=4.363$ arc-seconds. As can be seen in the figure, a good agreement can be found between $P_{\text {pos_ } i}$ and $P_{\text {neg_i } i}$ after the compensation of the influence of $\beta$. 
(a)

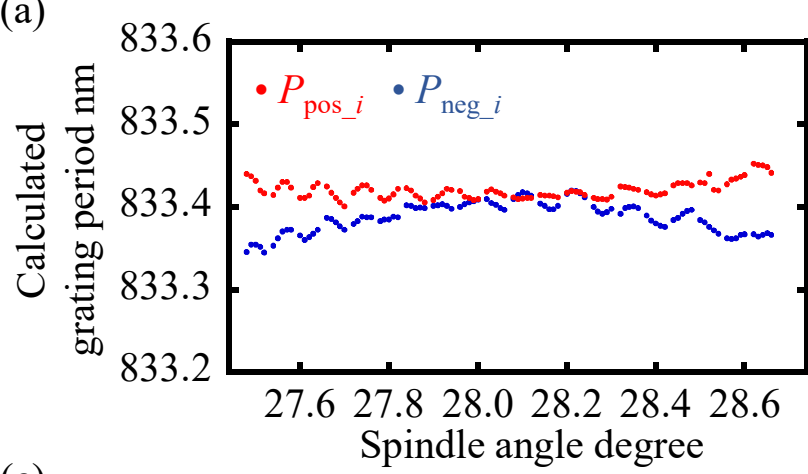

(c)

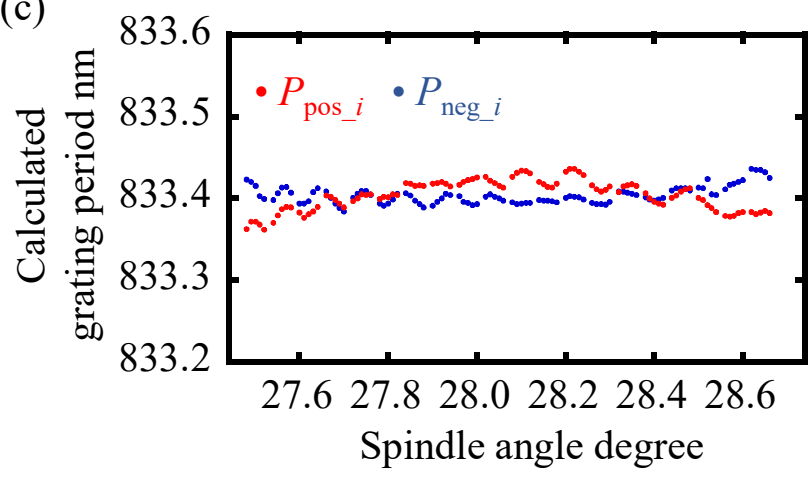

(b)

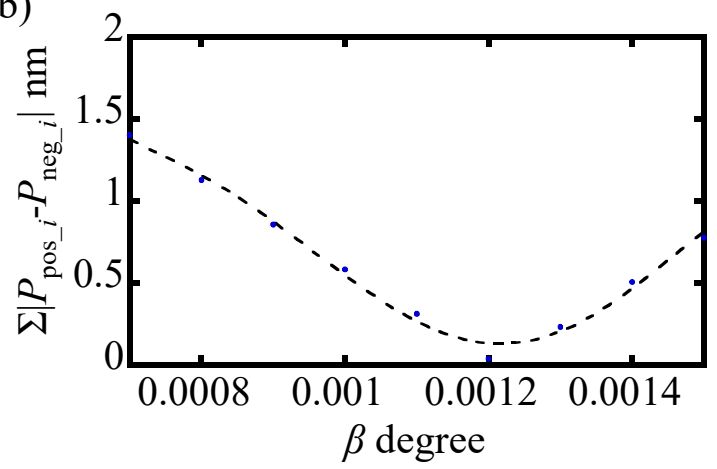

Figure 11. Grating pitch calculated from the peak wavelength obtained at each angular position of the scale grating: (a) Grating pitches $P_{\text {pos_ } i}$ and $P_{\text {neg } i} i$ calculated by using the positive and negative first-order diffracted beams without the compensation of $\beta(\beta=0)$; (b) Variation of $\Sigma\left|P_{\text {pos }_{-} i}-P_{\text {neg } \_} i\right|$ with the change in $\beta$; (c) $P_{\text {pos } \_i}$ and $P_{\text {neg } \_} i$ after the compensation of $\beta$ ( $\beta=4.363$ arc-seconds).

It should be noted that the optical frequency comb laser employed in this paper had a non-uniform spectrum, as can be seen in Figure 9. The non-uniform spectrum of the light source could affect the calculation of the peak wavelength based on the weight-of-mass method, resulting in the degradation of the uncertainty in pitch measurement. Compensation of the influence of the non-uniform spectrum of the mode-locked femtosecond laser beam employed in the setup was thus carried out by using the observed spectrum of the zeroth-order diffracted beam shown in Figure 9. In the compensation process, the spectrum of the first-order diffracted beam $I_{ \pm 1}\left(\lambda_{i}\right)$ obtained at each angular position of the spindle was divided by the spectrum of the zeroth-order diffracted beam $I_{0}\left(\lambda_{i}\right)$ to obtain the normalized spectrum $I_{\text {norm }}\left(\lambda_{i}\right)$. Figure $12 \mathrm{a}, \mathrm{b}$ shows the grating period $P_{\text {neg } \_i}$ calculated by using the spectra $I_{-1}\left(\lambda_{i}\right)$ and $I_{\text {norm }}\left(\lambda_{i}\right)$ of 100 optical modes observed in experiments, respectively. By the compensation process, the deviation of the calculated grating period $P_{\text {neg } \_i}$ obtained from the spectra in the wavelength range of $770 \mathrm{~nm}$ to $800 \mathrm{~nm}$ was reduced from $94.76 \mathrm{pm}$ to $74.34 \mathrm{pm}$. This result demonstrated the effectiveness of the compensation process for the reduction of the uncertainty of pitch measurement. It should be noted that a slight difference can still be observed between $P_{\text {pos } \_i}$ and $P_{\text {neg } \_} ;$i The possible root causes contain the influence of the chromatic aberration of the optical components in the setup, as well as the instability of the spectrum of the mode-locked femtosecond laser beam, and further detailed investigation is required for the better understanding of the results. 

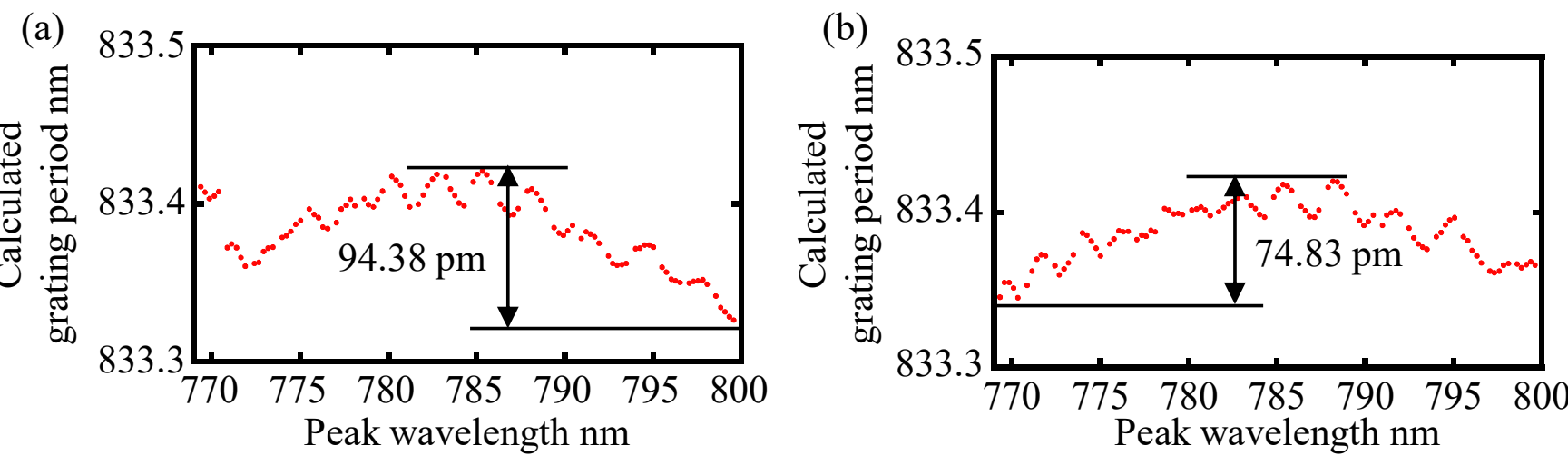

Figure 12. Compensation of the influence of the measurement laser beam spectrum. (a) Grating pitch calculated by using the spectra of the first-order diffracted beam $I_{-1}\left(\lambda_{i}\right) ;(\mathbf{b})$ Grating pitch calculated by using the normalized spectra $I_{\text {norm }}\left(\lambda_{i}\right)$.

\section{Uncertainty Analysis}

Measurement uncertainty analysis is also carried out based on the guide to the expression of uncertainty in measurement (GUM) [23] to verify the feasibility of the proposed method with the employment of an optical frequency comb laser. Since the mean of pitch values evaluated from multiple angles of diffraction of diffracted modes is evaluated in the proposed method, at first, the uncertainty of evaluating the pitch by a specific diffracted mode is evaluated. Then, the probability distribution of pitches from the angles of diffraction of entire modes was obtained to evaluate the measurement uncertainty of the proposed method.

Equations (4) and (5) can be modified as follows by considering the refractive index of air $n_{\text {air: }}$

$$
\begin{aligned}
& P_{\text {pos } \_i}=\frac{\lambda_{\text {pos } \_i}}{2 n_{\text {air }} \cos \left(\frac{\alpha-\beta}{2}\right) \sin \left(\theta_{\text {pos } \__{-} i}+\frac{\beta}{2}\right)} \\
& P_{\text {neg } \_i}=\frac{\lambda_{\text {neg } i} i}{2 n_{\text {air }} \cos \left(\frac{\alpha-\beta}{2}\right) \sin \left(\theta_{\text {neg } \_} i-\frac{\beta}{2}\right)}
\end{aligned}
$$

In the following, the contribution of each parameter in the equations is evaluated.

(a) Angular misalignment $\alpha$ of the measurement laser beam about the $Z$-axis with respect to the $X$-axis $(u(\alpha))$

At first, the contribution of $\alpha$ was evaluated. As described in the previous section of this paper, $\alpha$ was evaluated by using the zeroth-order diffracted beam reflected from the retroreflector; the $Y$-directional displacement of the focused laser beam on the CCD image sensor was converted into the angle $\alpha$ based on the principle of laser autocollimation [22]. Denoting the $Y$-positions of the focused laser beam on the image sensor when measuring the reflected beam from the retroreflector and the zeroth-order diffracted beam from the diffraction grating as $Y_{\text {retro }}$ and $Y_{0}$, respectively, Equation (6) can be rewritten as follows:

$$
\alpha=\arctan \left(\frac{Y_{\text {retro }}-Y_{0}}{F}\right)
$$

Then, the standard uncertainty $u(\alpha)$, which is the contribution of $\alpha$, can be evaluated by the following equation:

$$
u_{\alpha}=\sqrt{\left[c_{Y_{\text {retro }}} \cdot u_{Y_{\text {retro }}}\right]^{2}+\left[c_{Y_{0}} \cdot u_{Y_{0}}\right]^{2}+\left[c_{F} \cdot u_{F}\right]^{2}}
$$


where $u_{Y \text { retro, }} u_{Y 0}$ and $u_{F}$ are the standard uncertainties of $Y_{\text {retro }}, Y_{0}$ and $F$, respectively, while $c_{\text {Yretro }}, c_{Y 0}$ and $c_{F}$ are the sensitivity coefficients of $Y_{\text {retro }}, Y_{0}$ and $F$, respectively. Table 1 summarizes the contributions of each parameter on $u_{\alpha}$. The $Y$-position of the focused retroreflected beam and zeroth-order beam on the profiler was obtained as a mean of 200 repetitive trials with a standard deviation of $0.333 \mu \mathrm{m}$ and $0.299 \mu \mathrm{m}$, respectively. From Equation (10), the sensitivity coefficients $c_{Y \text { retro, }} c_{0}$ and $c_{Y}$ can be derived as follows:

$$
\begin{gathered}
c_{Y_{\text {retro }}}=\frac{\partial \alpha}{\partial Y_{\text {retro }}}=\frac{\partial}{\partial Y_{\text {retro }}}\left[\arctan \left(\frac{Y_{\text {retro }}-Y_{0}}{F}\right)\right]=\frac{1}{F} \cdot \frac{1}{1+\left(\frac{Y_{\text {retro }}-Y_{0}}{F}\right)^{2}} \\
c_{0}=\frac{\partial \alpha}{\partial Y_{0}}=\frac{\partial}{\partial Y_{0}}\left[\arctan \left(\frac{Y_{\text {retro }}-Y_{0}}{F}\right)\right]=\frac{-1}{F} \cdot \frac{1}{1+\left(\frac{Y_{\text {retro }}-Y_{0}}{F}\right)^{2}} \\
c_{F}=\frac{\partial \alpha}{\partial F}=\frac{\partial}{\partial F}\left[\arctan \left(\frac{Y_{\text {retro }}-Y_{0}}{F}\right)\right]=\frac{1}{1+\left(\frac{Y_{\text {retro }}-Y_{0}}{F}\right)^{2}} \cdot \frac{Y_{0}-Y_{\text {retro }}}{F^{2}}
\end{gathered}
$$

\begin{tabular}{|c|c|c|c|c|c|c|c|c|}
\hline Sources of Uncertainty & Symbol & Type & $\begin{array}{l}\text { Uncertainty } \\
\text { Value }\end{array}$ & $\begin{array}{l}\text { Probability } \\
\text { Distribution }\end{array}$ & Divisor & $\begin{array}{c}\text { Standard } \\
\text { Uncertainty } \\
u\end{array}$ & $\begin{array}{c}\text { Sensitivity } \\
\text { Coefficient } \\
c\end{array}$ & $|c| \cdot|u|$ \\
\hline $\begin{array}{l}\text { Repeatability of the } \\
\text { detection of } Y_{\text {retro }}\end{array}$ & $u_{\text {dretro }}$ & $\mathrm{A}$ & $0.333 \mu \mathrm{m}$ & Gaussian & $\sqrt{200}$ & $0.0235 \mu \mathrm{m}$ & $\begin{array}{c}0.116 \text { arc- } \\
\text { second } / \mu \mathrm{m}\end{array}$ & $\begin{array}{c}2.731 \times 10^{-3} \\
\text { arc-second }\end{array}$ \\
\hline $\begin{array}{l}\text { Repeatability of the } \\
\text { detection of } Y_{0}\end{array}$ & $u_{d 0 t h}$ & A & $0.299 \mu \mathrm{m}$ & Gaussian & $\sqrt{200}$ & $0.0211 \mu \mathrm{m}$ & $\begin{array}{l}-0.116 \text { arc- } \\
\text { second } / \mu \mathrm{m}\end{array}$ & $\begin{array}{c}2.448 \times 10^{-3} \\
\text { arc-second }\end{array}$ \\
\hline $\begin{array}{l}\text { Focal misalignment of the } \\
\text { image sensor with respect } \\
\text { to the objective lens }\end{array}$ & $u_{F}$ & B & $\pm 0.5 \mathrm{~mm}$ & Rectangular & $\sqrt{3}$ & $0.289 \mathrm{~mm}$ & $\begin{array}{l}-4.193 \times 10^{-3} \\
\text { arc- } \\
\text { second } / \mathrm{mm}\end{array}$ & $\begin{array}{c}1.211 \times 10^{-3} \\
\text { arc-second }\end{array}$ \\
\hline \multicolumn{8}{|c|}{$u(\alpha)$} & $\begin{array}{l}3.862 \times 10^{-3} \\
\text { arc-second }\end{array}$ \\
\hline
\end{tabular}

Table 1. Uncertainty of the angular misalignment $\alpha$.

By applying mean values of $Y_{\text {retro }}(-186.160 \mu \mathrm{m}), Y_{0}(-187.303 \mu \mathrm{m})$ and a nominal value of $F$ of $31.1 \mathrm{~mm}, c_{Y}$ retro, $c_{0}$ and $c_{Y}$ were evaluated as shown in Table 1 . It should be noted that the misalignment of the image sensor with respect to the objective lens was considered to be within $\pm 0.5 \mathrm{~mm}$. By evaluating the contribution of each uncertainty source, $u(\alpha)$ was evaluated to be $3.862 \times 10^{-3}$ arcsecond.

(b) Angular misalignment $\beta$ of the detector unit about the $X$-axis with respect to the Z-axis $(u(\beta))$

The contribution of $\beta$ was then estimated. As shown in Figure $11 \mathrm{~b}, \Sigma\left|P_{\text {pos } \_i}-P_{\text {neg } \_i}\right|$ corresponding to the change of $\beta$ in a step of $0.0001^{\circ}$ was calculated, then $\beta$ which minimize $\Sigma\left|P_{\text {pos } \_i}-P_{\text {neg } \_i}\right|$ was selected by taking the extremum of the data curve obtained by sixth-order polynomial approximation fitting. However, as shown in Figure $11 \mathrm{c}, P_{\text {pos }} i$ and $P_{\text {neg } \_i}$ did not perfectly match by this fitting process, and $\mid P_{\text {pos } \_i}-P_{\text {neg } \_} i$ at each angular position of the spindle could contribute to the uncertainty $u(\beta)$ of evaluating $\beta$ by polynomial approximation fitting. The deviation of $\beta_{i}$, which was obtained by the $i$ th positive and negative Littrow angles was evaluated as shown in Figure 13. A standard deviation of $\left|\beta-\beta_{\mathrm{i}}\right|$ was evaluated to be 1.875 arc-second. Since, 100 sets of $P_{\text {pos } \_} i$ and $P_{\text {neg }} i$ were employed, by applying a divisor of $\sqrt{100}, u(\beta)$ was evaluated to be 0.1875 arc-second. 


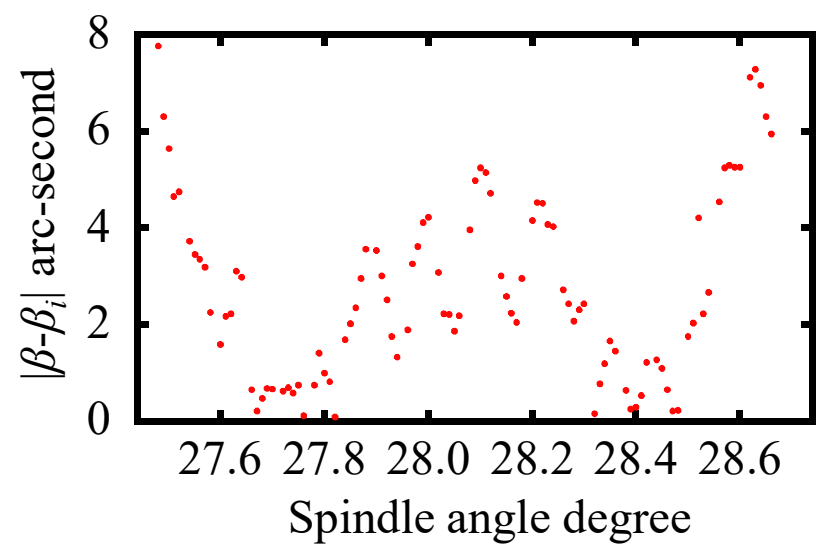

Figure 13. The deviation of $\beta_{i}$ from $\beta$.

(c) Uncertainty of the detection of the angle $\theta_{i}$ of the $i$ th-mode positive or negative first-order diffracted beam $\left(u\left(\theta_{i}\right)\right)$

In the proposed method, the $i$ th-mode first-order Littrow angle $\theta_{i}$ was obtained from the readings of the optical rotary encoder embedded to the precision air-bearing spindle. Therefore, the resolution, stability and uncertainty of the reading of the optical rotary encoder could contribute to the uncertainty $u\left(\theta_{i}\right)$ of detecting the $i$ th-mode first-order Littrow angle of the positive or negative first-order diffracted beam. The contribution of the resolution $\left(u_{\text {res }}\right)$ was estimated from the information in the specification sheet of the rotary encoder provided by the encoder manufacturer. Meanwhile, the contribution of the stability of the encoder reading $\left(u_{\mathrm{stb}}\right)$ could somehow be affected by the stability of the developed setup, and was then evaluated by using experimental results. As can be seen in Figure $6 a, b$, standard deviations of the encoder reading when the scale grating was kept stationary at the angular positions of the positive and negative first-order Littrow configuration were evaluated to be 0.06054 arc-second and 0.06475 arc-second, respectively. Since a mean of 200 encoder readings at each angular position of the grating was employed as $\theta_{i}$, by applying a divisor of $\sqrt{200}$ to the worse case ( 0.06475 arc-second), $u_{\mathrm{stb}}$ was evaluated to be $4.579 \times 10^{-3}$ arc-second. Table 2 summarizes the contribution of each uncertainty source to $u\left(\theta_{i}\right)$.

Table 2. Uncertainty of the detection of the $i$ th positive or negative first-order Littrow angle $\theta_{i}$.

\begin{tabular}{|c|c|c|c|c|c|c|c|c|}
\hline Sources of Uncertainty & Symbol & Type & $\begin{array}{l}\text { Uncertainty } \\
\text { Value }\end{array}$ & $\begin{array}{c}\text { Probability } \\
\text { Distribution }\end{array}$ & Divisor & $\begin{array}{c}\text { Standard } \\
\text { Uncertainty } \\
u\end{array}$ & $\begin{array}{c}\text { Sensitivity } \\
\text { Coefficient } \\
\qquad c\end{array}$ & $|c| \cdot|u|$ \\
\hline $\begin{array}{c}\text { Stability of the angular } \\
\text { output }\end{array}$ & $u_{\mathrm{stb}}$ & A & $\begin{array}{l}0.06475 \\
\text { arc-second }\end{array}$ & Gaussian & $\sqrt{200}$ & $\begin{array}{l}4.579 \times 10^{-3} \\
\text { arc-second }\end{array}$ & 1 & $\begin{array}{l}4.579 \times 10^{-3} \\
\text { arc-second }\end{array}$ \\
\hline $\begin{array}{l}\text { Resolution of rotary } \\
\text { encoder }\end{array}$ & $u_{r e s}$ & B & $\begin{array}{l}0.0038 \\
\text { arc-second }\end{array}$ & Rectangular & $\sqrt{3}$ & $\begin{array}{l}2.194 \times 10^{-3} \\
\text { arc-second }\end{array}$ & 1 & $\begin{array}{l}2.194 \times 10^{-3} \\
\text { arc-second }\end{array}$ \\
\hline \multicolumn{8}{|c|}{$u\left(\theta_{i}\right)$} & $\begin{array}{c}5.077 \times 10^{-3} \\
\text { arc-second }\end{array}$ \\
\hline
\end{tabular}

(d) Uncertainty of the light wavelength detected at each Littrow configuration $(u(\lambda i))$

In the proposed method, the light wavelength $\lambda_{\text {pos_i } i}$ or $\lambda_{\text {neg } i} i$ for pitch measurement is evaluated based on Equation (7) as the peak wavelength of the obtained spectrum. Therefore, light intensity fluctuation at each light wavelength and the uncertainty of the reading in the optical spectrum analyzer could contribute to the uncertainty $u\left(\lambda_{i}\right)$ of the light wavelength detected at each Littrow configuration. In this study, a value $\left(7.10 \times 10^{-4} \mathrm{~nm}\right)$ from the previous work by the authors' team [24] was employed as $u\left(\lambda_{i}\right)$.

(e) Uncertainty of the refractive index in air nair (u(nair))

The contribution of $n_{\text {air }}$ was then estimated. Since the experiment had been conducted in a laboratory environment, the contributions of the atmospheric pressure, air temperature, 
and humidity in the room atmospheric range should be considered. It should be noted that a lot of work has been made so far to evaluate the contribution of $n_{\text {air }}$. In this study, taking a value from the literature [25-28], $u\left(n_{\text {air }}\right)$ was estimated to be $\pm 5 \times 10^{-8}$ [26].

Regarding the contribution of each uncertainty source, standard uncertainties $u_{\mathrm{P} i}$ of the pitch $P_{i}$ obtained by the $i$ th positive or negative first-order Littrow angle can be estimated by the following equations:

$$
u_{P i}=\sqrt{(c(\alpha) \cdot u(\alpha))^{2}+(c(\beta) \cdot u(\beta))^{2}+\left(c\left(\theta_{i}\right) \cdot u\left(\theta_{i}\right)\right)^{2}+\left(c\left(\lambda_{i}\right) \cdot u\left(\lambda_{i}\right)\right)^{2}+\left(c\left(n_{\text {air }}\right) \cdot u\left(n_{\text {air }}\right)\right)^{2}}
$$

where $c(\alpha), c(\beta), c\left(\theta_{i}\right), c\left(\lambda_{i}\right)$ and $c\left(n_{\text {air }}\right)$ are the sensitivity coefficients that can be described by the following equations:

$$
\begin{gathered}
c(\alpha)=\frac{\lambda_{\mathrm{i}} \sin \left(\frac{\alpha-\beta}{2}\right)}{4 n_{\text {air }} \cos ^{2}\left(\frac{\alpha-\beta}{2}\right) \sin \left(\theta_{i}+\frac{\beta}{2}\right)} \\
c(\beta)=\frac{-\lambda_{\mathrm{i}}\left[\cos \left(\theta_{i}+\frac{\beta}{2}\right) \cos \left(\frac{\alpha-\beta}{2}\right)+\sin \left(\theta_{i}+\frac{\beta}{2}\right) \sin \left(\frac{\alpha-\beta}{2}\right)\right]}{4 n_{\text {air }}\left[\cos \left(\frac{\alpha-\beta}{2}\right) \sin \left(\theta_{i}+\frac{\beta}{2}\right)\right]^{2}} \\
c\left(\theta_{i}\right)=\frac{-\lambda_{\mathrm{i}} \cos \left(\theta_{i}+\frac{\beta}{2}\right)}{2 n_{\text {air }} \cos \left(\frac{\alpha-\beta}{2}\right) \sin ^{2}\left(\theta_{i}+\frac{\beta}{2}\right)} \\
c\left(\lambda_{i}\right)=\frac{1}{2 n_{\text {air }} \cos \left(\frac{\alpha-\beta}{2}\right) \sin \left(\theta_{i}+\frac{\beta}{2}\right)} \\
c\left(n_{\text {air }}\right)=\frac{-\lambda_{i}}{2 n_{\text {air }}{ }^{2} \cos \left(\frac{\alpha-\beta}{2}\right) \sin \left(\theta_{i}+\frac{\beta}{2}\right)}
\end{gathered}
$$

In the above equations, $\alpha$ is the angular misalignment of the measurement laser beam about the $Z$-axis, $\beta$ is the angular misalignment value of the measurement laser beam about the $X$-axis, $\theta_{i}$ is the average Littrow angle, $\lambda_{i}$ is the central wavelength, and $n_{\text {air }}$ is the refractive index in air. Table 3 summarizes the contribution of each uncertainty source. Based on Equation (15), $u_{P i}$ was evaluated to be $40.65 \mathrm{pm}$.

Table 3. Combined uncertainty.

\begin{tabular}{ccccc}
\hline $\begin{array}{c}\text { Sources of } \\
\text { Uncertainty }\end{array}$ & Symbol & Standard Uncertainty & Sensitivity Coefficient & $|\boldsymbol{c}| \cdot|\boldsymbol{u}| \mathbf{p m}$ \\
\hline$\alpha$ & $u(\alpha)$ & $\boldsymbol{u}$ & $\boldsymbol{c}$ & $3.484 \times 10^{-6}$ \\
$\beta$ & $u(\beta)$ & 0.187 arc-second & $-0.217 \mathrm{~nm} /$ arc-second & 40.58 \\
$\theta_{i}$ & $u\left(\theta_{i}\right)$ & $5.077 \times 10^{-3}$ arc-second & $-0.434 \mathrm{~nm} /$ arc-second & 2.203 \\
$\lambda_{i}$ & $u\left(\lambda_{i}\right)$ & $7.100 \times 10^{-4} \mathrm{~nm}$ & 1.062 & 0.754 \\
$n_{\text {air }}$ & $u\left(n_{\text {air }}\right)$ & $5.0 \times 10^{-8}$ & $-833.263 \mathrm{~nm}$ & $4.166 \times 10^{-2}$ \\
\hline & & Combined uncertainty $u_{P i}$ & & $40.65 \mathrm{pm}$ \\
\hline
\end{tabular}

An expanded uncertainty $U$ of the pitch was thus evaluated to be $81.30 \mathrm{pm}(k=2$, $95 \%$ confidence). From the results shown in Figure 12b, the pitch values were found to distribute in a range from $833.404 \pm 0.0372 \mathrm{~nm}$; the experimental results were found to be within the uncertainty value estimated in the above analysis. It should be noted that $U$ estimated in the above analysis is the expanded uncertainty of the pitch by a specific optical mode in the optical frequency comb laser. By using $N$ optical modes ( $N=100$ in Figure 12) observed in experiments, an expanded uncertainty of the pitch measurement by the proposed method becomes $81.30 / \sqrt{\mathrm{N}} \mathrm{pm}$. 


\section{Conclusions}

For the improvement of the accuracy of pitch measurement of a diffraction grating based on the laser diffraction with an optical frequency comb laser source, major modifications have been made to the optical setup and signal processing. Instead of employing a Fabry-Pérot etalon for the separation of the optical modes in the spectrum of the captured first-order diffracted beams, a weight of mass method has been employed in the signal processing to find out the peak wavelength in the spectrum for the calculation of the grating pitch. In addition, a mathematical model of the pitch measurement in the proposed method with the employment of the optical frequency comb laser has been established while considering misalignments of optical components in the setup. Experiments have been carried out to compensate for the misalignments of the optical components considered in the mathematical model. In addition, the influence of the non-uniform spectrum of the mode-locked femtosecond laser source employed in the developed setup has successfully been reduced through the normalization operations of the light spectra obtained by the detector unit in the setup. Furthermore, by using the developed mathematical model, the theoretical analysis of the uncertainty in the pitch measurement by the proposed method has been carried out based on the guide to the expression of uncertainty in measurement (GUM). Through the theoretical evaluation based on GUM, an expanded uncertainty $U$ of the pitch measurement of a diffraction grating with a nominal pitch density of $/ 1200 \mathrm{~mm}$ by using the information from a specific mode has been evaluated to be $81.30 \mathrm{pm}(k=2.95 \%$ confidence). The experimental results were found to be within the uncertainty value estimated in the above analysis. By using $N$ optical modes observed in experiments, an expanded uncertainty of the pitch measurement by the proposed method can be reduced to $81.30 / \sqrt{\mathrm{N}} \mathrm{pm}$. These results of the theoretical investigation and experiments have demonstrated the feasibility of the proposed pitch measurement with an optical frequency comb laser.

Author Contributions: Conceptualization, W.G. and Y.S.; methodology, D.W.S., L.Q., Y.C., Y.S. and W.G.; software, D.W.S., L.Q. and Y.S.; validation, Y.S., W.G. and H.M.; formal Analysis, D.W.S., L.Q., Y.S. and W.G.; investigation, D.W.S., L.Q., Y.C. and Y.S.; resources, Y.S. and W.G.; data Curation, Y.S. and W.G; Writing-Original Draft Preparation, D.W.S. and Y.S.; Writing-Review and Editing, W.G., Y.S. and E.M.; Visualization, W.G. and Y.S.; Supervision, W.G.; Project Administration, W.G.; Funding Acquisition, W.G., Y.S. and H.M. All authors have read and agreed to the published version of the manuscript.

Funding: This work is supported by the Japan Society for the Promotion of Science (JSPS) $20 \mathrm{H} 00211$.

Institutional Review Board Statement: Not applicable.

Informed Consent Statement: Not applicable.

Data Availability Statement: The data presented in this study are available on request from the corresponding author.

Acknowledgments: Dong Wook Shin would like to thank the Graduate Program for Integration of Mechanical Systems (GP-Mech) of Tohoku University, Japan.

Conflicts of Interest: The authors declare no conflict of interest. The funders had no role in the design of the study; in the collection, analyses, or interpretation of data; in the writing of the manuscript or in the decision to publish the results.

\section{References}

1. Gao, W.; Kim, S.W.; Bosse, H.; Haitjema, H.; Chen, Y.L.; Lu, X.D.; Knapp, W.; Weckenmann, A.; Estler, W.T.; Kunzmann, H. Measurement technologies for precision positioning. CIRP Ann.-Manuf. Technol. 2015, 64, 773-796. [CrossRef]

2. Lin, J.; Guan, J.; Wen, F.; Tan, J. High-resolution and wide range displacement measurement based on planar grating. Opt. Commun. 2017, 404, 132-138. [CrossRef]

3. Ye, W.; Zhang, M.; Zhu, Y.; Wang, L.; Hu, J.; Li, X.; Hu, C. Real-time displacement calculation and offline geometric calibration of the grating interferometer system for ultra-precision wafer stage measurement. Precis. Eng. 2019, 60, 413-420. [CrossRef] 
4. Dai, G.; Koenders, L.; Pohlenz, F.; Dziomba, T.; Danzebrink, H.U. Accurate and traceable calibration of one-dimensional gratings. Meas. Sci. Technol. 2005, 16, 1241-1249. [CrossRef]

5. Dai, G.; Pohlenz, F.; Dziomba, T.; Xu, M.; Diener, A.; Koenders, L.; Danzebrink, H.U. Accurate and traceable calibration of two-dimensional gratings. Meas. Sci. Technol. 2007, 18, 415-421. [CrossRef]

6. Misumi, I.; Gonda, S.; Huang, Q.; Keem, T.; Kurosawa, T.; Fujii, A.; Hisata, N.; Yamagishi, T.; Fujimoto, H.; Enjoji, K.; et al. Sub-hundred nanometre pitch measurements using an AFM with differential laser interferometers for designing usable lateral scales. Meas. Sci. Technol. 2005, 16, 2080-2090. [CrossRef]

7. Chen, J.; Liu, J.; Wang, X.; Zhang, L.; Deng, X.; Cheng, X.; Li, T. Optimization of nano-grating pitch evaluation method based on line edge roughness analysis. Meas. Sci. Rev. 2017, 17, 264-268. [CrossRef]

8. Brasil, D.A.; Alves, J.A.P.; Pekelsky, J.R. An imaging grating diffractometer for traceable calibration of grating pitch in the range $20 \mu \mathrm{m}$ to $350 \mathrm{~nm}$. J. Phys. Conf. Ser. 2015, 648, 012013. [CrossRef]

9. Pekelsky, J.R.; Eves, B.J.; Nistico, P.R.; Decker, J.E. Imaging laser diffractometer for traceable grating pitch calibration. Meas. Sci. Technol. 2007, 18, 375-383. [CrossRef]

10. Buhr, E.; Michaelis, W.; Diener, A.; Mirandé, W. Multi-wavelength VIS/UV optical diffractometer for high-accuracy calibration of nano-scale pitch standards. Meas. Sci. Technol. 2007, 18, 667-674. [CrossRef]

11. Sheng, B.; Chen, G.; Huang, Y.; Luo, L. Measurement of grating groove density using multiple diffraction orders and one standard wavelength. Appl. Opt. 2018, 57, 2514. [CrossRef] [PubMed]

12. Xie, Y.F.; Jia, W.; Zhao, D.; Ye, Z.H.; Sun, P.; Xiang, C.C.; Wang, J.; Zhou, C. Traceable and long-range grating pitch measurement with picometer resolution. Opt. Commun. 2020, 476, 126316. [CrossRef]

13. Xiong, X.; Matsukuma, H.; Shimizu, Y.; Gao, W. Evaluation of the pitch deviation of a linear scale based on a self-calibration method with a Fizeau interferometer. Meas. Sci. Technol. 2020, 31, 094002. [CrossRef]

14. Gao, W.; Haitjema, H.; Fang, F.Z.; Leach, R.K.; Cheung, C.F.; Savio, E.; Linares, J.M. On-machine and in-process surface metrology for precision manufacturing. CIRP Ann. 2019, 68, 843-866. [CrossRef]

15. Cundiff, S.T.; Fortier, T.M.; Ye, J.; Hall, J.L. Carrier-envelope phase stabilization of femtosecond modelocked lasers and direct optical frequency synthesis. In Proceedings of the Conference on Lasers and Electro-Optics, Postconference Technical Digest (IEEE Cat. No.01CH37170), Baltimore, MD, USA, 11 May 2001; p. 130.

16. Leopardi, H.; Davila-Rodriguez, J.; Quinlan, F.; Olson, J.; Sherman, J.A.; Diddams, S.A.; Fortier, T.M. Single-branch Er:fiber frequency comb for precision optical metrology with 10^-18 fractional instability. Optica 2017, 4, 879. [CrossRef]

17. Shimizu, Y.; Uehara, K.; Matsukuma, H.; Gao, W. Evaluation of the grating period based on laser diffraction by using a mode-locked femtosecond laser beam. J. Adv. Mech. Des. Syst. Manuf. 2018, 12, 1-10. [CrossRef]

18. Chen, Y.-L.; Shimizu, Y.; Tamada, J.; Kudo, Y.; Madokoro, S.; Nakamura, K.; Gao, W. Optical frequency domain angle measurement in a femtosecond laser autocollimator. Opt. Express 2017, 25, 16725. [CrossRef]

19. Korotkov, V.I.; Pulkin, S.A.; Vitushkin, A.L.; Vitushkin, L.F. Laser interferometric diffractometry for measurements of diffraction grating spacing. Appl. Opt. 1996, 35, 4782. [CrossRef] [PubMed]

20. Decker, J.E.; Eves, B.J.; Pekelsky, J.R.; Douglas, R.J. Evaluation of uncertainty in grating pitch measurement by optical diffraction using Monte Carlo methods. Meas. Sci. Technol. 2011, 22, 027001. [CrossRef]

21. Shimizu, Y.; Matsukuma, H.; Gao, W. Optical angle sensor technology based on the optical frequency comb laser. Appl. Sci. 2020, 10, 4047. [CrossRef]

22. Gao, W.; Saito, Y.; Muto, H.; Arai, Y.; Shimizu, Y. A three-axis autocollimator for detection of angular error motions of a precision stage. CIRP Ann.-Manuf. Technol. 2011, 60, 515-518. [CrossRef]

23. ISO Evaluation of measurement data-Guide to the expression of uncertainty in measurement. Int. Organ. Stand. Geneva ISBN 2008, 50, 134.

24. Sato, R.; Chen, C.; Matsukuma, H.; Shimizu, Y.; Gao, W. A new signal processing method for a differential chromatic confocal probe with a mode-locked femtosecond laser. Meas. Sci. Technol. 2020, 31, 094004. [CrossRef]

25. Owens, J.C. Optical Refractive Index of Air: Dependence on Pressure, Temperature and Composition. Appl. Opt. 1967, 6, 51. [CrossRef] [PubMed]

26. Peck, E.R.; Reeder, K. Dispersion of Air. J. Opt. Soc. Am. 1972, 62, 958-962. [CrossRef]

27. Ciddor, P.E. Refractive index of air: New equations for the visible and near infrared. Appl. Opt. 1996, 35, 1566. [CrossRef] [PubMed]

28. Birch, K.P.; Downs, M.J. An updated Edlén equation for the refractive index of air. Metrologia 1993, 30, 155-162. [CrossRef] 